\title{
Burkitt lymphoma-associated network construction and important network motif analysis
}

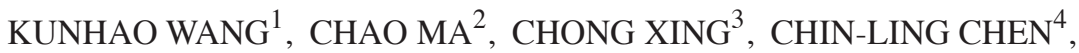 \\ ZHIGANG CHEN $^{1}$, YUXIA YAO ${ }^{1}$, JIANAN WANG ${ }^{1}$ and CHUNYU TAO ${ }^{1}$
}

\begin{abstract}
${ }^{1}$ School of Information Engineering, Changchun University of Science and Technology, Changchun, Jilin 130600; ${ }^{2}$ College of Digital Media, Shenzhen Institute of Information Technology, Shenzhen, Guangdong 518172; ${ }^{3}$ Department of Information and Technology, Changchun Finance College, Changchun, Jilin 130028, P.R. China; ${ }^{4}$ Department of Computer Science and Information Engineering, Chaoyang University of Technology, Taichung 41349, Taiwan, R.O.C.
\end{abstract}

Received August 15, 2016; Accepted May 11, 2018

\section{DOI: $10.3892 / \mathrm{ol} .2018 .9010$}

\begin{abstract}
Biological and medical researchers have discovered numerous transcription factors (TFs), microRNAs (miRNAs) and genes associated with Burkitt lymphoma (BL) through individual experiments; however, their regulatory mechanisms remain unclear. In the present study, BL-dysregulated and BL-associated networks were constructed to investigate these mechanisms. All data and regulatory associations were from known data resources and literature. The dysregulated network consisted of dysregulated TFs, miRNAs and genes, and partially determined the pathogenesis mechanisms underlying $\mathrm{BL}$. The BL-associated network consisted of BL-associated TFs, miRNAs and genes. It has been indicated that the network motif consisted of TFs, miRNAs and genes serve potential functions in numerous biological processes within cancer. Two of the most studied network motifs are feedback loop (FBL) and feed-forward loop (FFL). The important network motifs were extracted, including the FBL motif, 3-nodes FFL motif and 4-nodes motif, from BL-dysregulated and BL-associated networks, and 10 types of motifs were identified from BL-associated network. Finally, 26/31 FBL motifs, 45/75 3-nodes FFL motifs and 54/94 4-nodes motifs were obtained from the dysregulated/associated networks. A total of four TFs (E2F1, NFKB1, E2F4 and TCF3) exhibit complicated regulation associations in BL-associated networks. The
\end{abstract}

Correspondence to: Mr. Chao Ma, College of Digital Media, Shenzhen Institute of Information Technology, 2188 Dragon Avenue, Longgang, Shenzhen, Guangdong 518172, P.R. China

E-mail: billmach@163.com

Abbreviations: miRNA, microRNA; TFs, transcription factors; targets, target genes; BL, Burkitt lymphoma; NCBI, National Center for Biotechnology Information; TFBSs, transcription factor binding sites

Key words: Burkitt lymphoma, microRNA, transcription factor, network motif, host gene biological network does not demonstrate the dysregulated status for healthy people. When the individual becomes unwell, their biological network exhibits a dysregulated status. If the dysregulated status is regulated to a normal status by a number of medical methods, the diseases may be treated successfully. BL-dysregulated networks serve important roles in pathogenesis mechanisms underlying BL regulation of the dysregulated network, which may be an effective strategy that contributes to gene therapy for BL.

\section{Introduction}

Burkitt lymphoma (BL) is a highly aggressive, mature, B-cell lymphoma, and a type of non-Hodgkin lymphoma (NHL), and is associated with the Epstein-Barr virus (EBV) and oncogene (MYC) (1). BL is diagnosed in between 30 and 70 children per million and accounts for $82 \%$ of all NHLs in children and adolescents in equatorial African countries (2). The highest incidence rates of $\mathrm{BL}$ are located in tropical African countries, including Malawi, Uganda, Nigeria and Niger, with other African countries having notably lower incidence rates, which are similar to those of highly developed countries (3).

Transcription factors (TFs) and microRNAs (miRNA) are two types of key gene regulatory factors in multicellular genomes (4), which regulate gene expression at the transcriptional and post-transcriptional levels, respectively. TFs are proteins that bind to specific DNA sequences in the gene promoter region, activating or repressing gene expression $(5,6)$. miRNAs are endogenous, small, non-coding RNA molecules [ 22 nucleotides (nt) long], which silences mRNA expression via base-pairing with complementary sequences (7). They function as either tumor suppressors or oncogenes, and influence numerous cancer processes, including proliferation, differentiation and apoptosis $(8,9)$.

MiRNAs and TFs are key gene regulatory factors and can form certain network motifs (10). These motifs serve potential roles in numerous biological processes, including the occurrence and development of a number of diseases. For example, Li et al (11) identified that certain miRNA-TF synergistic regulatory motifs in human lung cancer influence proliferation and cell cycle of non-small cell lung cancer. Hsieh et al (12) 
identified that there are numerous crosstalking motif interconnections, and hypothesized that certain network motifs serve a function in cancer formation. Zhang et al (13) summarized the types of feed-back loops (FBLs) and feed-forward loops (FFLs), and reviewed their behaviors and functions according to biological processes and diseases. Among them, two of the most-studied network motifs are FBLs and FFLs. FBL is a motif where TFs and miRNAs regulate each other; whereas, FFL is a motif where TFs and miRNAs together regulate a common target gene, and TFs regulate miRNAs or miRNAs target TFs. FBL and FFL motifs have positive and negative roles in a number of biological processes with the FBL motif serving as a switch, for example 1sy- 6 and miR-273 contribute to a bistable switch in ASE neurons of Caenorhabdtis elegans (14), with the FFL motif serving as a buffer, for example miR-7 and Yan form coherent FFL motifs involved in the specification of photoreceptor cells in the Drosophila melanogaster eye (15). These motifs serve important roles in gene regulation in mammalians (16).

A number of miRNAs are coded from their associated genes, and the other miRNAs are coded from introns (17). In the transcription process, the majority of miRNAs are independently transcribed, whereas other miRNAs are transcribed together with their host gene (17), demonstrating a coupled regulation between miRNA and the protein-coding gene. A number of studies determined that intronic miRNA and its host gene are coordinately expressed, and they frequently function together in biological processes of diseases $(18,19)$.

Up to now, biological and medical researchers have discovered numerous TFs, miRNAs and genes associated with BL through individual experiments. Among them, the dysregulated miRNAs, TFs and genes serve important roles in various BL regulation processes processions, such as deregulation of the tumor protein 53 (TP53) pathway in pediatric BL, and increased expression of mouse double minute 4 human homolog of P53 binding protein could deregulate the TP53 pathway in paediatric BL cases (20). Additionally, the expression of miR-143 and miR-145 was consistently low in human BL cell lines, and thus they may be useful as biomarkers of B-cell malignancies (21). Dysregulated elements functioning together may result in the occurrence of BL; whereas, other non-dysregulated elements may serve smaller roles in BL, such as DUSP16, a novel, epigenetically-regulated determinant of c-Jun N-terminal kinase activation in BL (22), and miR-146a, the induction of which serves a role in the induction or maintenance of EBV latency in type III BL cells (23). Notably, BL-associated elements include BL-dysregulated elements.

Although these BL-associated elements were determined one by one, their regulatory mechanisms remain largely unknown. A previous study indicated that the occurrence of complex diseases is not the result of a single gene, but due to multiple genetic variants and environmental risk factors (24). In the present study, the focus was on BL-associated TFs, miRNAs and genes, as well as their validated regulatory associations $(\mathrm{TFs} \rightarrow$ miRNAs, TFs $\rightarrow$ genes, miRNAs $\rightarrow$ genes and host gene $\rightarrow$ miRNA; $\rightarrow$ represents regulatory relation, meaning that the former regulates the latter), in order to investigate their mechanisms in BL. All data were collected from public databases. In the present study, the dysregulated elements included TFs, miRNAs and genes that had:
Genetic mutations; deletions; abnormal expression; single nucleotide polymorphisms; inactivation; overexpression; low expression; downregulation; upregulation; or differential expression. The associated elements included dysregulated and non-dysregulated elements. Based on dysregulated and associated elements, the dysregulated and associated networks of BL were constructed separately, then the two networks were systematically investigated to determine the features and key network motifs (2-nodes FBL motifs, 3-nodes FFL motifs and 4-nodes motifs). Due to FFL motifs serving important roles in a number of diseases $(25,26)$, this type of motif was constructed with the P-match method (27), and it was combined with the $1,000 \mathrm{nt}$ promoter region sequences of dysregulated miRNA targets. The workflow of the present study was depicted in Fig. 1, and further information was described in the materials and methods. The understanding of the dysregulated network will improve the knowledge regarding the pathogenesis of $\mathrm{BL}$, and the understanding of the associated network will contribute to the prevention and therapy of BL.

\section{Materials and methods}

BL-associated genes. BL-associated genes were collected from the DisGeNET (version 4.0) (28), MalaCards (29), Phenopedia (30) and PubMed (http://www.ncbi.nlm.nih. gov/pubmed) databases. DisGeNET (28) is an open platform that integrates the information regarding the amount of gene-disease associations from a number of public data sources and literature. MalaCards (29) is a searchable database integrating human maladies and their annotations. Phenopedia (30) is a web-based application used to search for summarized information regarding human genetic associations with genes or diseases from PubMed. DisGeNET, MalaCards and Phenopedia provided relevant files regarding disease-genes, which were imported into a spreadsheet using Microsoft Excel 2010 (Microsoft Corporation, Redmond, WA, USA). In the PubMed database, the search terms 'Burkitt lymphoma' and 'gene' were used, and the date was set from 2010 to present. A large number of study abstracts were reviewed for BL-associated genes and these genes were also listed in the spreadsheet. Experimentally-validated data were divided into two types: Dysregulated and non-dysregulated genes. All symbols of genes were unified by the official symbols of the NCBI gene database (https://www.ncbi.nlm. nih.gov/gene). The unique gene IDs were used as key indexes and duplicated genes were removed.

BL-associated miRNAs. BL-associated miRNAs were collected from the Human microRNA Disease Database (HMDD; version 2.0) (31), miR2Disease (32), PhenomiR (33) and PubMed databases. HMDD (31) is a database containing experimentally-validated human miRNA-disease associations from original papers. miR2Disease (32) is a manually curated database providing a comprehensive resource regarding miRNA in various human diseases, by reviewing numerous published papers. PhenomiR (33) is a knowledgebase providing miRNA-disease and miRNA-biological processes associations from a number of studies. HMDD, miR2Disease and PhenomiR provided relevant data regarding disease-miRNAs which were imported into a spreadsheet using Microsoft Excel. 


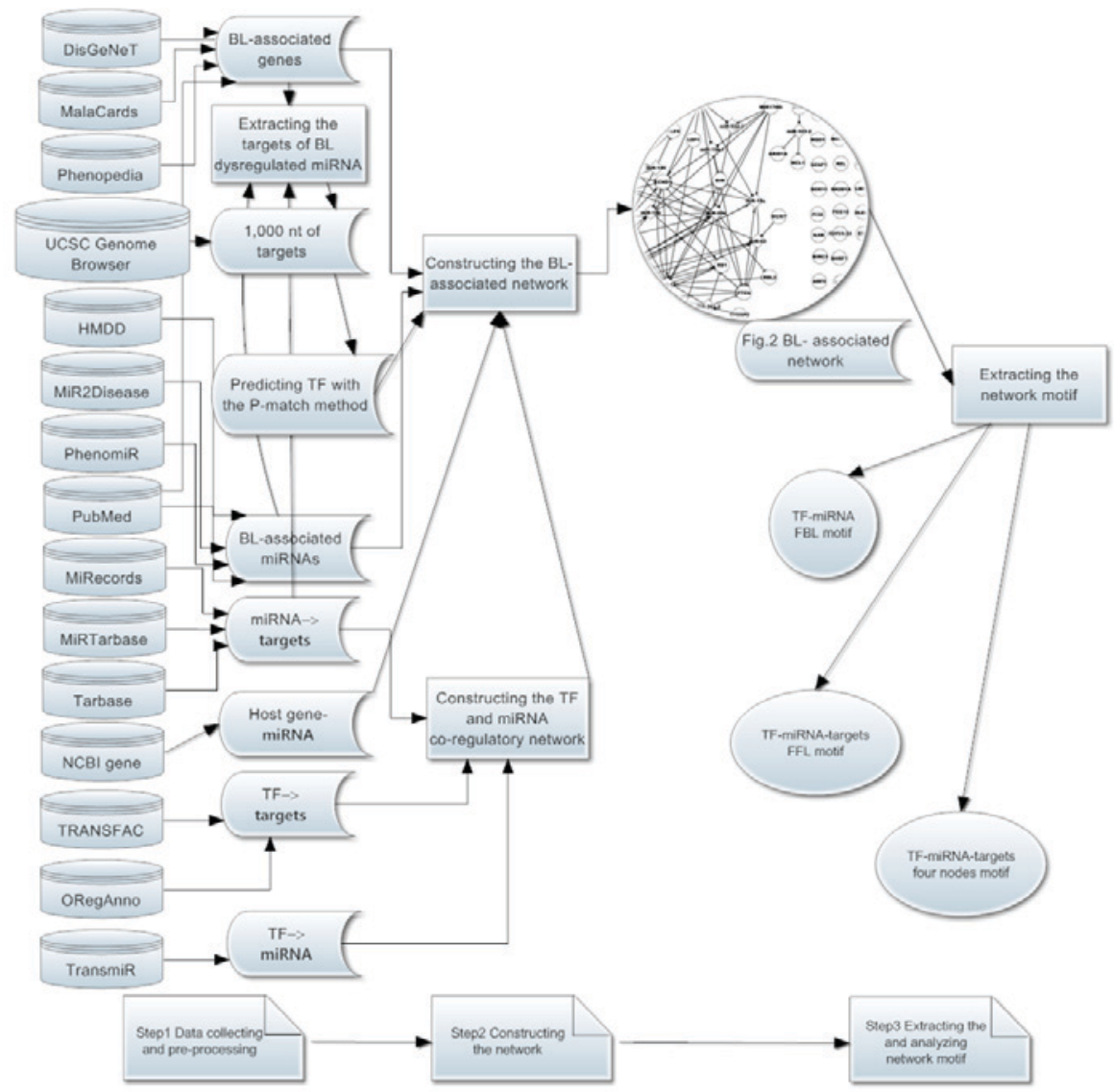

Figure 1. The workflow of the present study. (Step 1) Data collection and pre-processing, including BL-associated TFs, miRNAs, genes and their regulatory associations. (Step 2) Based on step 1 data, construction of the BL-dysregulated and BL-associated networks. (Step 3) Extracting and analyzing network motifs, including FBL, FFL and TF-miRNA-genes 4-nodes motifs. The arrow represents process flows. BL, Burkitt lymphoma; targets, target genes; TF, transcription factor; FBL, feed-back loop; FFL, feed-forward loop; nt, nucleotide; miRNA, microRNA.

In the PubMed database, the search terms 'Burkitt lymphoma' and 'miRNA' were used, and the date was set from 2010 to present. Numerous abstracts were read and any BL-associated miRNAs identified were also added to the spreadsheet. Experimentally-validated data were divided into dysregulated and non-dysregulated miRNAs. All symbols of miRNAs were unified by the official symbols of the NCBI gene database (https://www.ncbi.nlm.nih.gov/gene). The unique miRNA IDs were used as key indexes and duplicated miRNAs were removed.

TF-miRNA. TF-miRNA validated associations were collected from TransmiR (version 1.2) (34), a database containing $735 \mathrm{TF}$-miRNA regulatory pairs that were manually curated from published literature. TransmiR provides spreadsheet containing TF-miRNAs to the user.

TF-gene. TF-gene validated associations were collected from TRANScription FACtor (TRANSFAC; version 11.4) (35) and Open REGulatory ANNOtation (ORegAnno) (36) databases. TRANSFAC (35) is a database containing eukaryotic TFs, their genomic binding sites (TFBSs) and DNA-binding profiles. This database provides details on TFs and their features, DNA-binding sites and relevant targets, extracted from public literature. ORegAnno (36) is an open access database containing regulatory regions, TFBSs, RNA binding sites, regulatory variants and other regulatory elements. Data from ORegAnno are extracted from the literature and highly curated resources. TRANSFAC and ORegAnno provide data regarding TF-genes, which were imported into a spreadsheet using Microsoft Excel. All symbols of TFs and genes were unified by the official symbols of the NCBI gene database (https://www.ncbi.nlm.nih.gov/gene). The unique TF and gene IDs were used as key indexes and any duplications of regulatory associations between TFs and genes were removed.

Host gene of miRNA. Host gene-miRNA associations were collected from National Center for Biotechnology Information (NCBI) gene database (http://www.ncbi.nlm.nih.gov/gene/), a comprehensive gene database covering a wide range of species and including detailed gene information. Each BL-associated miRNA symbol was input into the search field of the NCBI gene database. In the genomic context section of each return page, host genes of miRNAs were extracted and placed in a spreadsheet using Microsoft Excel. All symbols of genes and miRNAs were unified by the official symbols of the NCBI gene database (https://www.ncbi.nlm.nih.gov/gene). The unique gene and miRNA IDs were used as key indexes and any duplications of association between miRNAs and genes were removed.

miRNA-gene. miRNA-gene validated associations were collected from Tarbase (version 7.0) (37), miRTarBase 


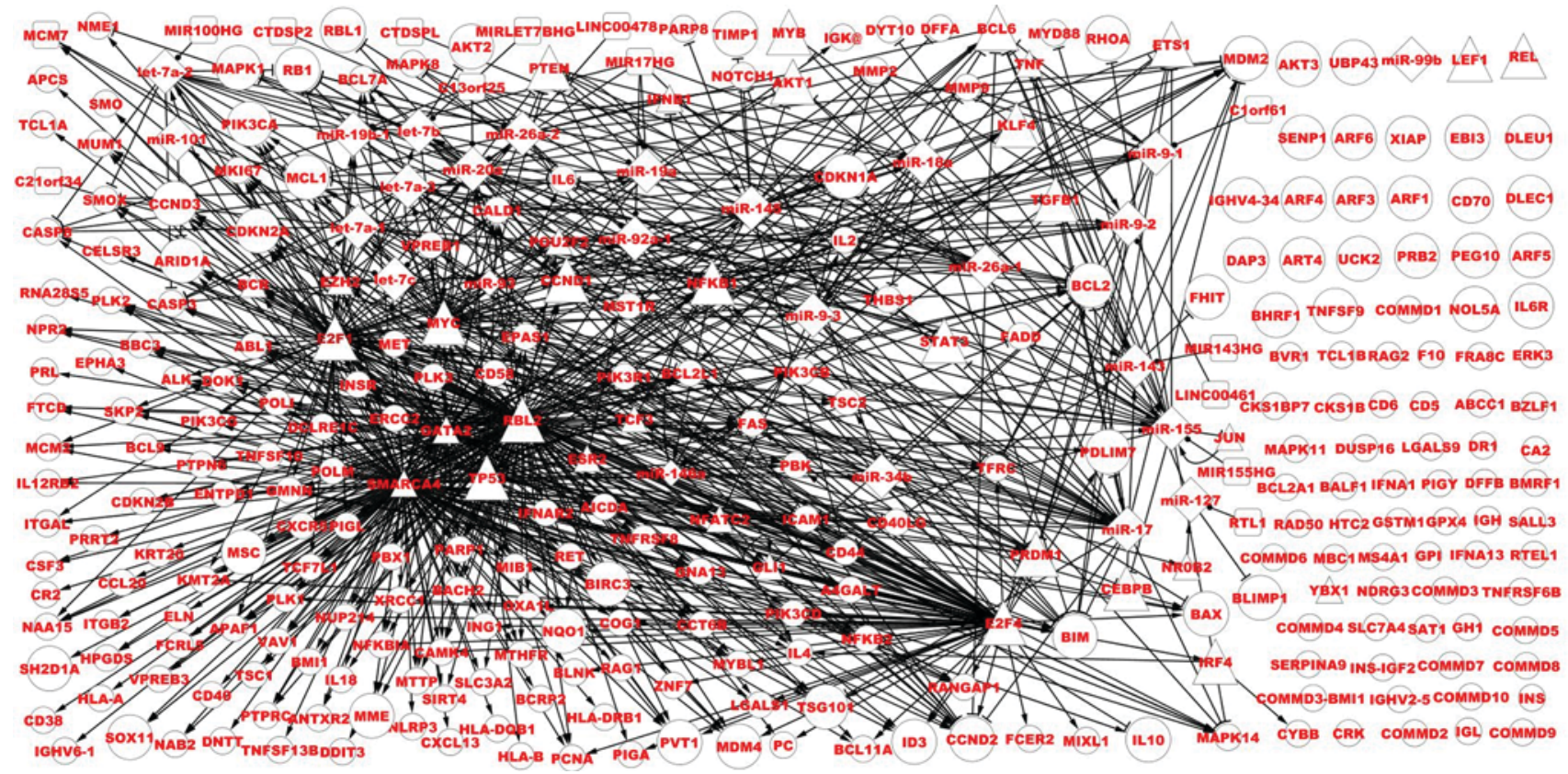

Figure 2. The BL-associated network. Bigger nodes represent dysregulated TFs, miRNAs and targets in BL, smaller nodes represent non-dysregulated and associated TFs, miRNAs and target genes in BL. Circles represent targets, triangles represent TFs, squares represent host genes and diamonds represent miRNAs. TF, transcription factor; miRNA, microRNA; BL, Burkitt lymphoma.

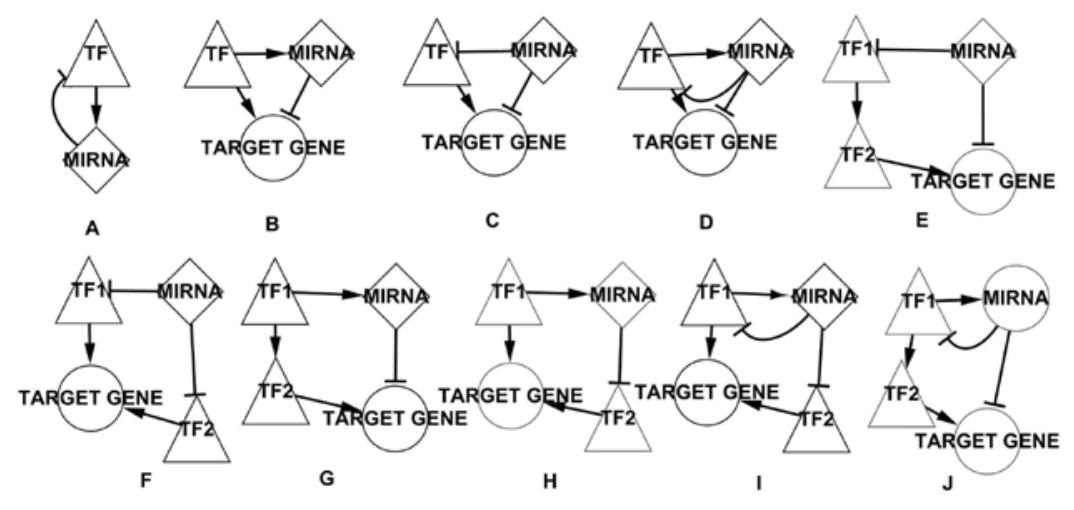

Figure 3. A number of network motifs in the BL-associated network. (A) The FBL motif. (B) The TF-regulated FFL motif. (C) The miRNA-regulated FFL motif. (D) The composite FFL motif. (E) The 4-nodes miRNA-regulated motif that miRNA and TF together regulate a target gene, for example miR-17, TCF3, GLI1 and CCND2 form this type of motif. (F) The 4-nodes miRNA-regulated motif that two TFs together regulate a target gene, for example miR-20, TP53, E2F1 and SMO form this type of motif. (G) The 4-nodes TF-regulated motif that miRNA and TF together regulate a target gene, for example miR-155, TP53, NFATC2 and ICAM1 form this type of motif. (H) The 4-nodes TF-regulated motif that two TFs together regulate a target gene, for example miR-155, TP53, BCL6 and BCL2L1 form this type of motif. (I) The 4-nodes composite motif that two TFs together regulate a target gene, for example miR-17, E2F1, RBL2 and POU2F2 form this type of motif. (J) The 4-nodes composite motif that miRNA and TF together regulate a target gene, for example let-7a, E2F1, RBL2 and CCND2 form this type of motif. Triangles represent TFs, diamonds represent miRNAs, circles represent target genes. FBL, feed-back loop; FFL, feed-forward loop; miRNA, microRNA; BL, Burkitt lymphoma; TF, transcription factor.

(version 6) (38) and miRecords (version 4) (39). Tarbase (37) is a manually curated database that contains 100,000 s of miRNA-gene pairs that have been experimentally identified. In this database, the focus is on regulatory pairs extracted by western blot analysis, northern blot analysis and quantitative PCR (qPCR). miRTarBase (38) also provides experimentally-validated miRNA-gene pairs. MiRTarBase (version 6.0) contains 7,439 strongly-validated miRNA-gene pairs and 348,007 miRNA-gene pairs from CLIP-seq. This database contains strongly-validated regulatory pairs extracted by western blot analysis, northern blot analysis, qPCR, and other reporter assays. MiRecords (39) is another resource containing animal miRNA-gene pairs. It contains validated targets from literature curation and predicted targets from 11 established miRNA target prediction programs. Tarbase, miRTarBase and miRecords provided data regarding miRNA-genes which were copied into a spreadsheet using Microsoft Excel. All symbols including TFs, miRNAs and genes were unified by the official symbol of the NCBI gene database. The unique TF, gene and miRNA IDs were used as key indexes and any duplications of associations between TFs, miRNAs and genes were removed.

Networks construction. Based on experimentally-validated regulatory data consisting of: TFs $\rightarrow$ miRNAs; TFs $\rightarrow$ genes; 
Table I. Statistical data regarding BL-associated network.

BL-associated data

Data source

Number

BL-associated genes

BL-associated miRNAs

BL-associated TF-miRNA pairs

BL-associated TF-gene pairs

BL-associated host gene-miRNA pairs

BL-associated miRNA-gene pairs
DisGeNET, MalaCards, Phenopedia PubMed, P-match

HMDD, miR2Disease, PhenomiR, PubMed

25

TransmiR

64

TransFAC ORegAnno

534

NCBI gene database

24

Tarbase miRTarbase miRecords

miRNA, microRNA; TF, transcription factor; BL, Burkitt lymphoma.

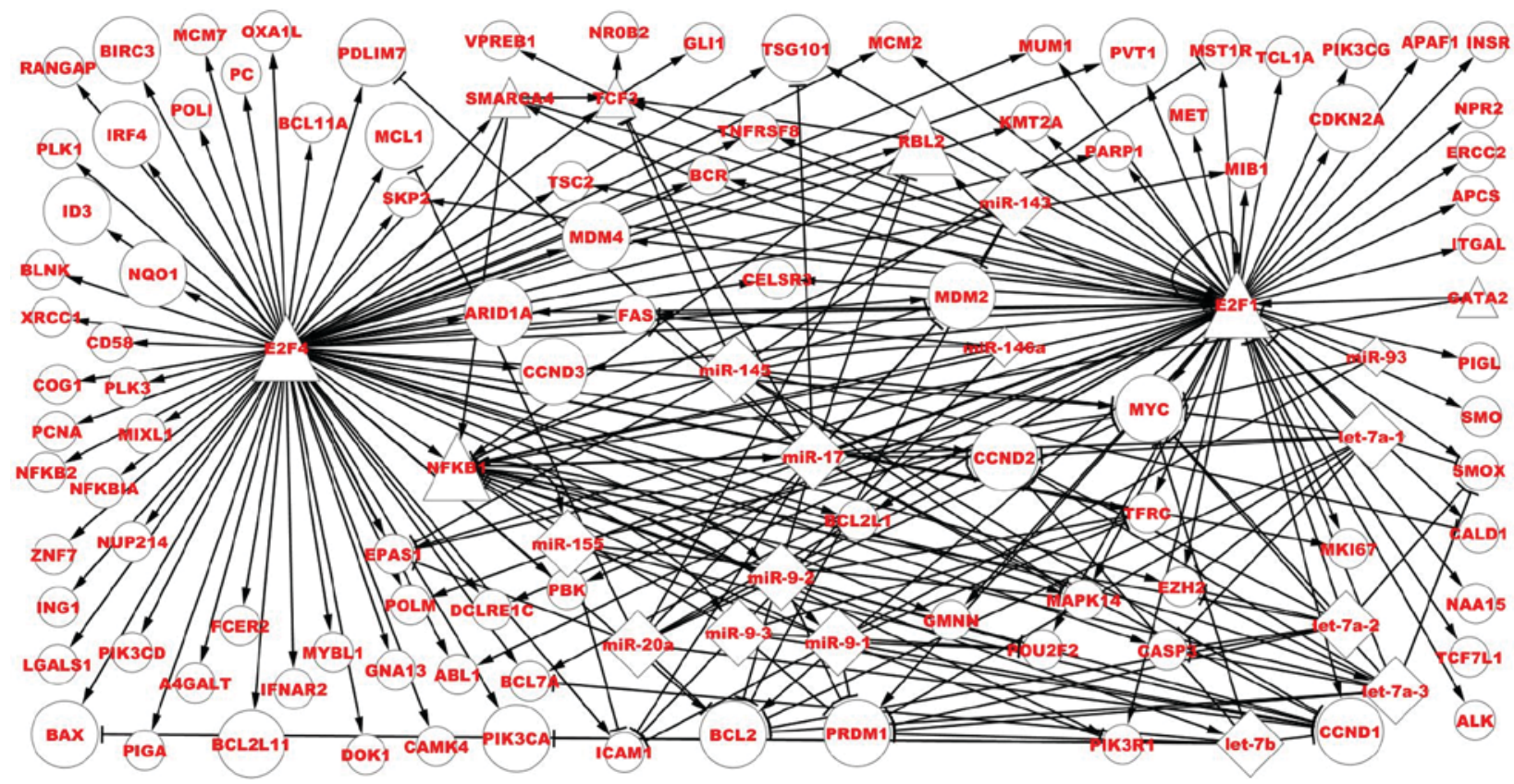

Figure 4. Direct regulatory associations of E2F1, NFKB1,E2F4 and TCF3 in the BL-associated network. Bigger nodes represent dysregulated TFs, miRNAs and targets, smaller nodes represent associated and non-dysregulated TFs, miRNAs and target genes. Circles represent targets, triangles represent TFs and diamonds represent miRNAs. TF, transcription factors; E2F1, E2F TF1; NFKB1, nuclear factor кB subunit 1; TCF3, TF3; miR, microRNA; BL, Burkitt lymphoma.

miRNAs $\rightarrow$ genes; and host gene $\rightarrow$ miRNAs, the TFs and miRNAs co-regulatory network was constructed by combining these regulatory pairs. This network was named the validated regulatory network.

Based on BL-associated data consisting of TFs, miRNAs and their host gene, genes, and the validated regulatory network, the BL-associated network was constructed. Firstly, theses nodes were mapped onto the validated regulatory network. Secondly, these regulatory data was extracted to construct the BL-associated regulatory network. Lastly, the BL-associated regulatory network and other BL-associated data, that did not contain regulatory associations with other TFs, genes and miRNAs, were combined into a network that was designated the BL-associated network (Fig. 2).

According to the features of FBLs and FFLs network motifs described in the introduction, network motifs were manually extracted from the BL-associated network. A total of 10 types of motif were identified (Fig. 3).

In total, 4 TFs (E2F1, NFKB1, E2F4 and TCF3) exhibited complicated regulatory relations in the BL-associated network, with maximum in- and out-degrees, which alongside miRNAs form network motifs (Fig. 4).

Using a similar method that was used to construct the BL-dysregulated network, the dysregulated data (TFs, miRNAs and genes in BL) was mapped onto the validated regulatory network and these regulatory data was extracted to construct the BL-dysregulated regulatory network. Lastly, the network and other BL-dysregulated data, that did not contain regulatory associations with other TFs, genes and miRNAs, were combined into the called BL-dysregulated network. The software (version 2.8.2) (40) was used to visualize the network. Dysregulated expression was present in three modes: Upregulation, downregulation and no expression.

Table I presents statistical data regarding BL-associated network. BL-associated data include associated genes, miRNAs, TF-miRNA pairs, TF-gene pairs, host gene-miRNA pairs and miRNA-gene pairs. BL-associated genes were collected from five data sources and 320 genes were identified. BL-associated miRNAs were collected from four data sources and 25 miRNAs were identified. BL-associated TF-miRNA 
Table III. Statistical data of key network motifs in BL-dysregulated and BL-associated networks.

\begin{tabular}{ccc}
\hline Dysregulated & Associated \\
notif type & network & network
\end{tabular}

Number of 3-nodes FFLs

miRNA-regulated FFLs

TF-regulated FFLs

Composite FFLs

Number of 4-nodes motifs miRNA-regulated motifs TF-regulated motifs

Composite motifs

Number of 2-nodes FBLs

26

miRNA, microRNA; TFs, transcription factors; FFL, feed-forward loop; FBL, feed-back loop; BL, Burkitt lymphoma.

pairs were collected from TransmiR and 64 pairs were identified. BL-associated TF-gene pairs were collected from two data sources and 534 pairs were identified. BL-associated host gene-miRNA pairs were collected from the NCBI gene database and 24 pairs were identified. BL-associated miRNA-gene pairs were collected from three data sources and 256 pairs were identified.

According to the type of nodes and type of regulatory associations, statistical data, including number of nodes and edges from BL-associated network and BL-dysregulated network, were manually extracted (Table II).

Predicted FFL motif. The predicted FFL motifs were constructed with the following method. Firstly, the 1,000 nt promoter region sequences of BL-associated genes targeted by dysregulated miRNAs were downloaded from UCSC (41); subsequently, the regulatory relations between the aforementioned dysregulated miRNAs and aforementioned targets in the BL-associated network were focused on and miRNA-gene pairs were extracted from the BL-associated network. Secondly, TFs of the aforementioned targets were predicted with the 1,000 nt promoter region sequences of the genes and the P-match method, and then the TF-gene pairs were obtained. The P-Match method is able to identify TFBSs in DNA sequences, and includes a matrix library as well as sets of known TFBSs collected from TRANSFAC. In the present study, the vertebrate matrixes and restricted high quality matrixes were used. The high quality criterion denotes the following: The threshold rate for a false negative result was set at 50\% (threshold value is 0.5 ), and a threshold was also set to differentiate positive from false positive results. Matrixes which produce the highest number of false positive results are defined as low quality. Thirdly, the focus was only on the predicted TFs included in transmiR and BL-associated miRNAs; subsequently, the corresponding TF-miRNA pairs were extracted from transmiR, and then the TF-miRNA pairs were obtained. Lastly, the TF-gene, TF-miRNA and miRNA-gene pairs were combined, and then FFL motifs were obtained. 
According to types of motifs, statistical data, including number of 3-nodes FFLs, number of 4-nodes motifs and number of 2-nodes FBLs, were manually extracted from the BL-associated and BL-dysregulated networks (Table III).

\section{Results}

BL-associated network. Fig. 2 depicts the BL-associated network, which includes dysregulated and non-dysregulated elements. Notably, the BL-associated network includes the dysregulated network. Table II displays the statistical data of the two networks. Although 14 host genes are not dysregulated, they may serve a role in the BL-associated processes. The dysregulated network partially reveals the pathogenesis of BL. The associated network not only partially demonstrated the regulatory mechanism underlying the occurrence of BL, but also determined a number of regulatory mechanisms associated with the prevention and therapy of BL.

The key network motifs were manually extracted from the BL-associated network. Fig. 3 depicts 10 types of key network motifs, consisting of TFs, miRNAs and targets in the BL-associated network. Fig. 3A depicts the FBL motif, a type of key regulator associated with cancer, in which TFs and miRNAs interact with each other. Fig. 3B depicts the TF-regulated FFL motif, where TFs and miRNAs together regulate a target gene, TFs regulate miRNAs and TFs serve as regulatory initiators. Fig. 3C depicts the miRNA-regulated FFL motif, where TFs and miRNAs together regulate a target gene, miRNAs regulate TFs and miRNAs serve as regulatory initiators. Fig. 3D depicts the composite FFL motif, where TFs and miRNAs together regulate a target gene and miRNAs and TFs regulate each other. Fig. $3 \mathrm{E}$ and $\mathrm{F}$ depict two types of 4 nodes miRNA-regulated motifs, in Fig. 3E, miRNAs regulate a target gene and a TF, which regulates another TF that regulates the target gene regulated by the miRNAs, in Fig. 3F, miRNAs regulate two TFs, which together regulate a target gene, and miRNAs serve as regulatory initiators. Fig. 3G and $\mathrm{H}$ depict two types of 4-nodes TF-regulated motifs, in Fig. 3G, TFs regulate a miRNA and a TF, which regulates a target gene regulated by the miRNA, in Fig. $3 \mathrm{H}$, TFs regulate a miRNA and a target gene, which is regulated by a TF regulated by the miRNA, and TFs serve as regulatory initiators, Fig. 3I and J depict two types of 4-nodes composite motifs, which are similar to the aforementioned two types (miRNA-regulated and TF-regulated) of 4-nodes motif, however, in these types the miRNAs and TFs regulate each other.

Table III displays statistical data regarding network motifs in dysregulated and associated networks of BL. The dysregulated network includes 45 3-nodes FFL motifs, 15 4-nodes motifs and 26 FBL motifs, whilst the associated network includes 75 3-nodes FFL motifs, 99 4-nodes motifs and 31 FBL motifs.

Regulatory network of predicted TF in the BL-associated network. Finally, 38 predicted TFs were obtained. Among these TFs, E2F TF1 (E2F1), nuclear factor $\kappa \mathrm{B}$ subunit 1 (NFKB1) and E2F4 are dysregulated genes, and TF3 (TCF3) is an associated and non-dysregulated gene. Fig. 4 depicts the direct regulatory associations of four TFs in the BL-associated network. E2F1 regulates 57 targets, and among them, 13 genes are dysregulated, with the others being non-dysregulated. E2F1 regulates 10 miRNAs, and among them, nine miRNAs are dysregulated and one miRNA is non-dysregulated, with five TFs regulating E2F1 and six miRNAs targeting E2F1. E2F1, its targets and these miRNAs form 38 FFLs and six FBLs. E2F4 regulates 79 targets, and among them, 21 genes are dysregulated, with the others being non-dysregulated. NFKB1 regulates eight miRNAs, which are all dysregulated, five TFs [E2F1, E2F4, GATA binding protein 2, RB transcriptional corepressor-like 2 (RBL2) and SMARCA4] regulate NFKB1, nine miRNAs target NFKB1 and six miRNAs form six FBLs. TCF3 regulates three targets (GLI family zinc finger 1, V-set pre-B cell surrogate light chain 1 and nuclear receptor subfamily 0 group B member 2), which are all non-dysregulated, four TFs (E2F1, E2F4, RBL2 and SMARCA4) regulate TCF3 and two miRNAs (miR-145 and miR-17) target TCF3.

\section{Discussion}

In the present study, the BL-dysregulated and BL-associated networks were constructed. These networks determined a portion of the regulatory mechanism of genes and miRNAs on BL. In the dysregulated network, all of the nodes had dysregulated expression, except a number of host genes that did not have dysregulated expression. The biological network does not exhibit a dysregulated status for healthy people. When an individual becomes unwell, their biological network demonstrates a dysregulated status. If the dysregulated status is regulated to a normal status by a number of medical methods, the diseases may be treated successfully. BL-dysregulated networks serve important roles in pathogenesis mechanisms underlying BL, and the regulation of the dysregulated network may be an effective strategy that contributes to gene therapy for BL.

In the dysregulated network, a number of single nodes, that do not have regulatory associations with other nodes, serve important roles in portions of the BL progression process, including apoptosis regulator BHRF1 which functions as a survival factor in Wp-restricted BL and rescued the cells from the apoptosis induced by dnEBNA1 expression (42), is deleted in lung and esophageal cancer 1, and is a modulator of proliferation, apoptosis and migration in BL (43). The mechanism underlying their influence on the progression of $\mathrm{BL}$ requiresfurther research. A number of dysregulatory-expressed nodes not only formed dysregulated regulatory pathways, such as $\mathrm{E} 2 \mathrm{~F} 1 \rightarrow \mathrm{MYC}$, TP53 $\rightarrow \mathrm{miR}-20 \mathrm{a} \rightarrow \mathrm{B}$-cell lymphoma 2 and $\mathrm{E} 2 \mathrm{~F} 4 \rightarrow \mathrm{MYC} \rightarrow$ miR-19b-1 $\rightarrow$ cyclin D1, but also form a number of key network motifs, such as FBL motifs (phosphatase and tensin homolog $\leftrightarrow \mathrm{miR}-19 \mathrm{a}$ and MYB $\leftrightarrow \mathrm{miR}-155), 3$-nodes FFL motif $(\mathrm{E} 2 \mathrm{~F} 1 \rightarrow \mathrm{BCL} 2$ and $\mathrm{E} 2 \mathrm{~F} 1 \rightarrow \mathrm{miR}-18 \mathrm{a} \rightarrow \mathrm{BCL} 2)$ and 4 -nodes motif $(\mathrm{miR}-17 \rightarrow \mathrm{RBL} 2 \rightarrow \mathrm{MYC}$ and miR-17 $\rightarrow$ TP53 $\rightarrow$ MYC). The mechanisms underlying the dysregulated regulatory pathways and network motifs influencing the occurrence, development and therapy of BL require further research in order to determine them.

\section{Acknowledgements}

Not applicable. 


\section{Funding}

The present study was supported by the 13th Five Year Plan of Science and Technology Research by Education Department of Jilin Province of China (grant no. 2016352), the 13th Five Year Plan of Science and Technology Research by Education Department of Jilin Province of China (grant no. JJKH20181369KJ), Scientific Research Project of Shenzhen Institute of Information Technology (grant no. QN201716) and the Scientific Research Staring Foundation of Changchun University of Science and Technology (grant no. 2016420).

\section{Availability of data and materials}

The datasets used and/or analyzed during the current study are available from the corresponding author on reasonable request.

\section{Authors' contributions}

$\mathrm{KW}$ wrote and revised the manuscript. KW, CLC, CM and CX conceived and designed the study and methodology. ZC and YY collected and preprocessed the data. JW and CT analyzed the data.

\section{Ethics approval and consent to participate}

Not applicable.

\section{Patient consent for publication}

Not applicable.

\section{Competing interests}

The authors declare that they have no competing interests.

\section{References}

1. Molyneux EM, Rochford R, Griffin B, Newton R, Jackson G, Menon G, Harrison CJ, Israels T and Bailey S: Burkitt's lymphoma. Lancet 379: 1234-1244, 2012.

2. Naresh KN, Raphael M, Ayers L, Hurwitz N, Calbi V, Rogena E, Sayed S, Sherman O, Ibrahim HA, Lazzi S, et al: Lymphomas in sub-Saharan Africa-what can we learn and how can we help in improving diagnosis, managing patients and fostering translational research? Br J Haematol 154: 696-703, 2011.

3. Stefan C, Bray F, Ferlay J, Liu B and Maxwell Parkin D: Cancer of childhood in sub-Saharan Africa. Ecancermedicalscience 11: 755,2017

4. Hobert O: Gene regulation by transcription factors and microRNAs. Science 319: 1785-1786, 2008.

5. Latchman DS: Transcription factors: An overview. Int J Biochem Cell Biol 29: 1305-1312, 1997.

6. Karin M: Too many transcription factors: Positive and negative interactions. New Biol 2: 126-131, 1990.

7. Ambros V: The functions of animal microRNAs. Nature 431: 350-355, 2004

8. Ha TY: MicroRNAs in human diseases: From cancer to cardiovascular disease. Immune Netw 11: 135-154, 2011.

9. Lee YS and Dutta A: MicroRNAs in cancer. Annu Rev Pathol 4: 199-227, 2009.

10. Su N, Wang Y, Qian M and Deng M: Combinatorial regulation of transcription factors and microRNAs. BMC Syst Biol 4: 150, 2010.
11. Li K, Li Z, Zhao N, Xu Y, Liu Y, Zhou Y, Shang D, Qiu F, Zhang $\mathrm{R}$, Chang $\mathrm{Z}$ and $\mathrm{Xu} \mathrm{Y}$ : Functional analysis of microRNA and transcription factor synergistic regulatory network based on identifying regulatory motifs in non-small cell lung cancer. BMC Syst Biol 7: 122, 2013.

12. Hsieh WT, Tzeng KR, Ciou JS, Tsai JJ, Kurubanjerdjit N, Huang $\mathrm{CH}$ and $\mathrm{Ng} \mathrm{KL}$ : Transcription factor and microRNA-regulated network motifs for cancer and signal transduction networks. BMC Syst Biol 9 (Suppl 1): S5, 2015.

13. Zhang HM, Kuang S, Xiong X, Gao T, Liu C and Guo AY: Transcription factor and microR NA co-regulatory loops: Important regulatory motifs in biological processes and diseases. Brief Bioinform 16: 45-58, 2015.

14. Hobert O: Architecture of a microRNA-controlled gene regulatory network that diversifies neuronal cell fates. Cold Spring Harb Symp Quant Biol 71: 181-188, 2006.

15. Li X, Cassidy JJ, Reinke CA, Fischboeck S and Carthew RW: A microRNA imparts robustness against environmental fluctuation during development. Cell 137: 273-282, 2009.

16. Tsang J, Zhu J and van Oudenaarden A: MicroRNA-mediated feedback and feedforward loops are recurrent network motifs in mammals. Mol Cell 26: 753-767, 2007.

17. Rodriguez A, Griffiths-Jones S, Ashurst JL and Bradley A: Identification of mammalian microRNA host genes and transcription units. Genome Res 14: 1902-1910, 2004.

18. Baskerville $\mathrm{S}$ and Bartel DP: Microarray profiling of microRNAs reveals frequent coexpression with neighboring miRNAs and host genes. RNA 11: 241-247, 2005.

19. Cao G, Huang B, Liu Z, Zhang J, Xu H, Xia W, Li J, Li S, Chen L, Ding H, et al: Intronic miR-301 feedback regulates its host gene, ska2, in A549 cells by targeting MEOX2 to affect ERK/CREB pathways. Biochem Biophys Res Commun 396: 978-982, 2010.

20. Leventaki V, Rodic V, Tripp SR, Bayerl MG, Perkins SL, Barnette P, Schiffman JD and Miles RR: TP53 pathway analysis in paediatric Burkitt lymphoma reveals increased MDM4 expression as the only TP53 pathway abnormality detected in a subset of cases. Br J Haematol 158: 763-771, 2012.

21. Akao Y, Nakagawa Y, Kitade Y, Kinoshita T and Naoe T: Downregulation of microRNAs-143 and -145 in B-cell malignancies. Cancer Sci 98: 1914-1920, 2007.

22. Lee S, Syed N, Taylor J, Smith P, Griffin B, Baens M, Bai M, Bourantas K, Stebbing J, Naresh K, et al: DUSP16 isan epigenetically regulated determinant of JNK signalling in Burkitt's lymphoma. Br J Cancer 103: 265-274, 2010.

23. Motsch N, Pfuhl T, Mrazek J, Barth S and Grässer FA: Epstein-Barr virus-encoded latent membrane protein 1 (LMP1) induces the expression of the cellular microRNA miR-146a. RNA Biol 4: 131-137, 2007.

24. Hirschhorn JN and Daly MJ: Genome-wide association studies for common diseases and complex traits. Nat Rev Genet 6: 95-108, 2005.

25. Qin S, Ma F and Chen L: Gene regulatory networks by transcription factors and microRNAs in breast cancer. Bioinformatics 31: 76-83, 2015.

26. Lin Y, Zhang Q, Zhang HM, Liu W, Liu CJ, Li Q and Guo AY: Transcription factor and miRNA co-regulatory network reveals shared and specific regulators in the development of $B$ cell and T cell. Sci Rep 5: 15215, 2015.

27. Chekmenev DS, Haid C and Kel AE: P-Match: Transcription factor binding site search by combining patterns and weight matrices. Nucleic Acids Res 33 (Web Server issue): W432-W437, 2005.

28. Piñero J, Queralt-Rosinach N, Bravo À, Deu-Pons J, Bauer-Mehren A, Baron M, Sanz F and Furlong LI: DisGeNET: A discovery platform for the dynamical exploration of human diseases and their genes. Database (Oxford): bav028, 2015.

29. Rappaport N, Nativ N, Stelzer G, Twik M, Guan-Golan Y, Stein TI, Bahir I, Belinky F, Morrey CP, Safran M and Lancet D: MalaCards: An integrated compendium for diseases and their annotation. Database (Oxford): bat018, 2013.

30. Yu W, Clyne M, Khoury MJ and Gwinn M: Phenopedia and genopedia: Disease-centered and gene-centered views of the evolving knowledge of human genetic associations. Bioinformatics 26: 145-146, 2010.

31. Li Y, Qiu C, Tu J, Geng B, Yang J, Jiang T and Cui Q: HMDD v2.0: A database for experimentally supported human microRNA and disease associations. Nucleic Acids Res 42 (Database Issue): D1070-D1074, 2014 
32. Jiang Q, Wang Y, Hao Y, Juan L, Teng M, Zhang X, Li M, Wang $\mathrm{G}$ and Liu Y: miR2Disease: A manually curated database for microRNA deregulation in human disease. Nucleic Acids Res 37 (Database Issue): D98-D104, 2009.

33. Ruepp A, Kowarsch A and Theis F: PhenomiR: microRNAs in human diseases and biological processes. Methods Mol Biol 822: 249-260, 2012.

34. Wang J, Lu M, Qiu C and Cui Q: TransmiR: A transcription factor-microRNA regulation database. Nucleic Acids Res 38 (Database Issue): D119-D122, 2010.

35. Wingender E, Chen X, Hehl R, Karas H, Liebich I, Matys V, Meinhardt T, Prüss M, Reuter I and Schacherer F: TRANSFAC: An integrated system for gene expression regulation. Nucleic Acids Res 28: 316-319, 2000

36. Lesurf R, Cotto KC, Wang G, Griffith M, Kasaian K, Jones SJ, Montgomery SB and Griffith OL; Open Regulatory Annotation Consortium: ORegAnno 3.0: A community-driven resource for curated regulatory annotation. Nucleic Acids Res 44 (D1): D126-D132, 2016

37. Vlachos IS, Paraskevopoulou MD, Karagkouni D, Georgakilas G, Vergoulis T, Kanellos I, Anastasopoulos IL, Maniou S, Karathanou K, Kalfakakou D, et al: DIANA-TarBase v7.0: Indexing more than half a million experimentally supported miRNA:mRNA interactions. Nucleic Acids Res 43 (Database Issue): D153-D159, 2015.

38. Chou CH, Chang NW, Shrestha S, Hsu SD, Lin YL, Lee WH, Yang CD, Hong HC, Wei TY, Tu SJ, et al: miRTarBase 2016: Updates to the experimentally validated miRNA-target interactions database. Nucleic Acids Res 44 (D1): D239-D247, 2016.
39. Xiao F, Zuo Z, Cai G, Kang S, Gao X and Li T: miRecords: An integrated resource for microRNA-target interactions. Nucleic Acids Res 37 (Database Issue): D105-D110, 2009.

40. Shannon P, Markiel A, Ozier O, Baliga NS, Wang JT, Ramage D, Amin N, Schwikowski B and Ideker T: Cytoscape: A software environment for integrated models of biomolecular interaction networks. Genome Res 13: 2498-2504, 2003.

41. Fujita PA, Rhead B, Zweig AS, Hinrichs AS, Karolchik D, Cline MS, Goldman M, Barber GP, Clawson H, Coelho A, et al: The UCSC Genome Browser database: Update 2011. Nucleic Acids Res 39 (Database Issue): D876-D882, 2011.

42. Watanabe A, Maruo S, Ito T, Ito M, Katsumura KR and Takada K: Epstein-Barr virus-encoded Bcl-2 homologue functions as a survival factor in Wp-restricted Burkitt lymphoma cell line P3HR-1. J Virol 84: 2893-2901, 2010.

43. Feng M, Huang B, Du Z, Xu X and Chen Z: DLC-1 as a modulator of proliferation, apoptosis and migration in Burkitt's lymphoma cells. Mol Biol Rep 38: 1915-1920, 2011.

This work is licensed under a Creative Commons Attribution-NonCommercial-NoDerivatives 4.0 International (CC BY-NC-ND 4.0) License. 